\title{
On the possible connection between galactic featureless-spectrum sources and stellar-mass black holes
}

\author{
L. Chmyreva ${ }^{1}$ and G. Beskin ${ }^{1}$ \\ Special Astrophysical Observatory, Nizhnij Arkhyz 369167, Russia
}

Received: July 31, 2019; Accepted: December 3, 2019

\begin{abstract}
The problem of direct detection of a black hole event horizon still remains unresolved. Isolated stellar-mass black holes could be ideal objects for solving this problem, since the event horizon would not be screened by the surrounding interstellar plasma due to its low accretion rate. We search for black hole candidates in their presumed birth locations, computed in the assumption that they could have originated in high-mass binaries. Theories predict that isolated black holes should exhibit featureless flat spectra covering the entire electromagnetic range. Objects classified as DC dwarfs (cool white dwarfs with featureless continuum spectra) are among such galactic sources, and they also constitute a good sample for searching for possible black hole candidates among them. We analyze sources with available spectral, photometric, and proper motion data. The final list contains 5 sources that fit the selection criteria.
\end{abstract}

\section{Introduction}

Direct detection of a black hole $(\mathrm{BH})$ event horizon is a problem that still remains largely unsolved. Searching for isolated stellar-mass black holes, spherically accreting interstellar medium (ISM) of low density, is of great importance, as such objects are ideal for detecting and studying the event horizon. The spectrum of such a black hole was first calculated by Shvartsman (1971). He demonstrated that an emitting halo of accreted matter forms around such objects and generates optical featureless emission. The majority of such emission comes from the regions near the horizon, at approximately $(3-5) r_{g}$, where $r_{g}$ is the Schwarzschild radius. It is therefore possible to observe the interaction of a $\mathrm{BH}$ with its surrounding environment.

An isolated stellar mass black hole would be subject to low-rate $\left(10^{-6}-10^{-9}\right.$ $\left.\dot{M}_{E d d}\right)$ spherical accretion of the ISM. It should exhibit synchrotron emission in the increased magnetic field if (1) the thermal electrons are in the range of $10^{14}-10^{16} \mathrm{~Hz}$ and $(2)$ the nonthermal ones are in the range of $10^{16}-10^{19} \mathrm{~Hz}$. This combination of both radiated components yields a practically flat spectrum from infrared to $\gamma$-rays. The hard spectral component is highly variable due to the emission of nonthermal electron blobs (for details, see Beskin \& Karpov 
2005, and references therein). For typical ISM parameters, a BH seen from a distance of $100 \mathrm{pc}$ would exhibit the following features:

$-16-25^{m}$ optical source

- highly variable bright X-ray counterpart

- variable component of optical emission $\sim 18-27^{m}$

- time scale of the variability $\sim 10^{-4} \mathrm{~s}$

- relative flare amplitudes $\sim 0.2-6 \%$ in various spectral bands

- proper motion.

Spherical accretion onto isolated stellar-mass black holes has been investigated by numerous authors, including early works of Zeldovich \& Novikov (1971); Novikov \& Thorne (1973); Shapiro (1973); Bisnovatyi-Kogan \& Ruzmaikin (1974); Meszaros (1975). The first solution to the spherical accretion problem was derived by Bondi (1952). Spectra for the resulting accretion flow were calculated by Ipser \& Price (1982) and McDowell (1985). Heckler \& Kolb (1996) proposed searching for isolated black holes using data from the Sloan Digital Sky Survey (SDSS). Their estimates used a spherical accretion model, and the method of Ipser \& Price (1982) was used to compute the spectrum. Chisholm et al. (2003) searched for nearby isolated BH using optical and X-ray data and reported the positions and colors of $55 \mathrm{BH}$ candidates and estimated their likelihood to be black holes.

As we mentioned above, theories predict that the main observational manifestation of a BH is a featureless flat spectrum (Shvartsman 1971; Beskin \& Karpov 2005). In this work, we seek sources with flat spectra and no significant spectral features over a large range of wavelengths, very fast variability, and luminosity within the predicted boundaries. Our search for possible candidates consists of two parts:

- a search restricted to specific regions in the sky as described below, and

- a general search involving an all-sky investigation of featureless-spectrum sources such as DC dwarfs (cool white dwarfs with helium-dominated atmospheres and featureless spectra with no lines) and BL Lacertae.

\section{Method}

In the first part, the sky regions where the search was performed are determined as follows. With at least $50 \%$ of stars known to be members of binary or multiple systems (Batten, 1967; Duquennoy \& Mayor, 1991; Halbwachs et al., 2003), it is safe to assume that many BHs, as well as relatively young neutron stars (NS) that we observe as radio pulsars, originated in high-mass binaries. In $\mathrm{BH}+\mathrm{NS}$ pairs, the more massive $\mathrm{BH}$ would have formed in the first supernova explosion in the system, which has a roughly $40 \%$ chance of staying gravitationally bound (Bethe \& Brown 1998). The second supernova explosion, in which a pulsar is born, generally disrupts the system. The high velocities pulsars obtained in the 
process (Hobbs et al. 2005) can be used to trace them back to their birthplaces, restricting therefore the $\mathrm{BH}$-candidate search area (Prokhorov \& Popov, 2002; Chmyreva et al., 2010).

In the second part, work on which is now in progress, the search for BH candidates is not restricted to specific regions, but is done throughout the whole sky. Such a search would concentrate on sources with already measured spectra available in public databases. Specifically, we are looking for sources with featureless flat spectra across the entire electromagnetic range, as described above and fitting the other criteria. To this end, we investigate the available catalogs for white dwarfs of DC spectral type, BL Lacertae objects that have not been confirmed and might be galactic sources with proper motions available in GAIA Data Release 2, and also any objects marked as "unknown" and whose spectra have no significant lines and could not be classified. A high signal-to-noise ratio is desired, since noisy spectra cannot be truly considered featureless. Such sources are then analyzed according to the other selection criteria described above.

\section{Candidate selection}

In order to identify the regions in the sky suitable for the search according to the first part of the method, we created an initial sample of 16 isolated radio pulsars with measured proper motions and parallaxes, relatively small spindown ages, and no previous associations that fit the criteria. To compute the sky-projected areas of their births, 100,000 trajectories were simulated for each pulsar, tracing them back according to their age estimates. The birthplaces of 4 youngest pulsars in the sample, J0139+5814, J0922+0638, J0358+5413, and $\mathrm{J} 1935+1616$, the first two of which are shown in Figure 1 as an example, have demonstrated the best results and were analyzed for possible objects of interest.

We used publicly available databases including but not limited to ROSAT, FERMI, XMM-Newton, FIRST, WISE, 2MASS, GALEX, SDSS, SIMBAD, ATNF, NED, LAMOST. First, we selected all non-optical sources that fall into the 3-sigma contours of the pulsar birth locations ( $5 \gamma$-ray sources, 250 X-ray sources, 1800 radio sources). These non-optical sources were then crossidentified with each other and with possible optical counterparts within their positional error ellipses. For this, we used the SDSS database (where available), and other catalogs otherwise. We constructed spectral energy distributions (SEDs) for the candidates with a hard-energy range component, and demonstrated that they are very similar to those of BL Lacertae objects. In addition to sources with a hard component, we also considered optical sources classified as blue objects and white dwarfs, as their observational manifestations are similar to those of the objects of interest. The spectra of 14 of the pre-selected candidates are shown in Figure 2. The spectrum of another candidate classified as a DC-type dwarf is shown in Figure 3. 

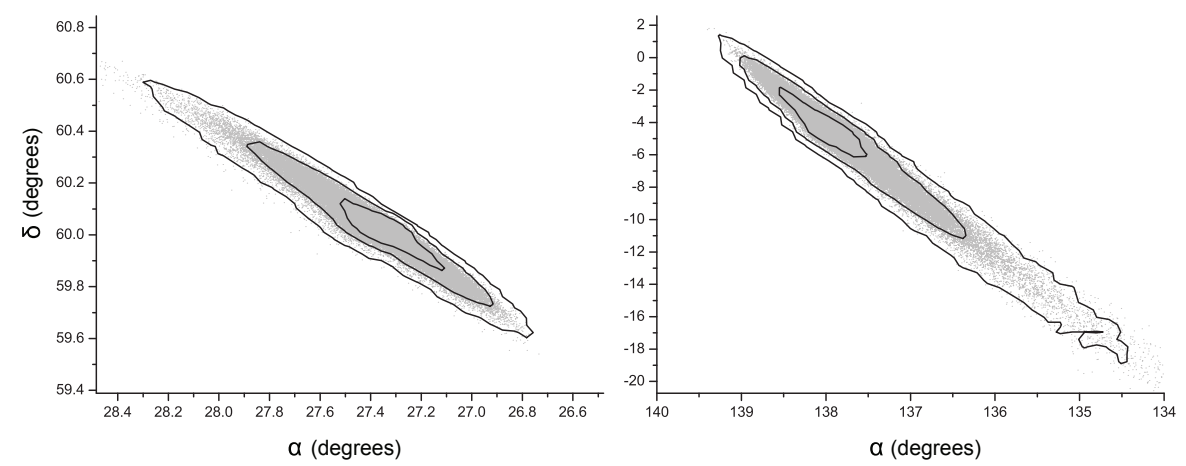

Figure 1. Simulated birthplaces of pulsars J0139+5814 (left) and J0922+0638 (right). The axes show the right ascension $(\alpha)$ and declination $(\delta)$.

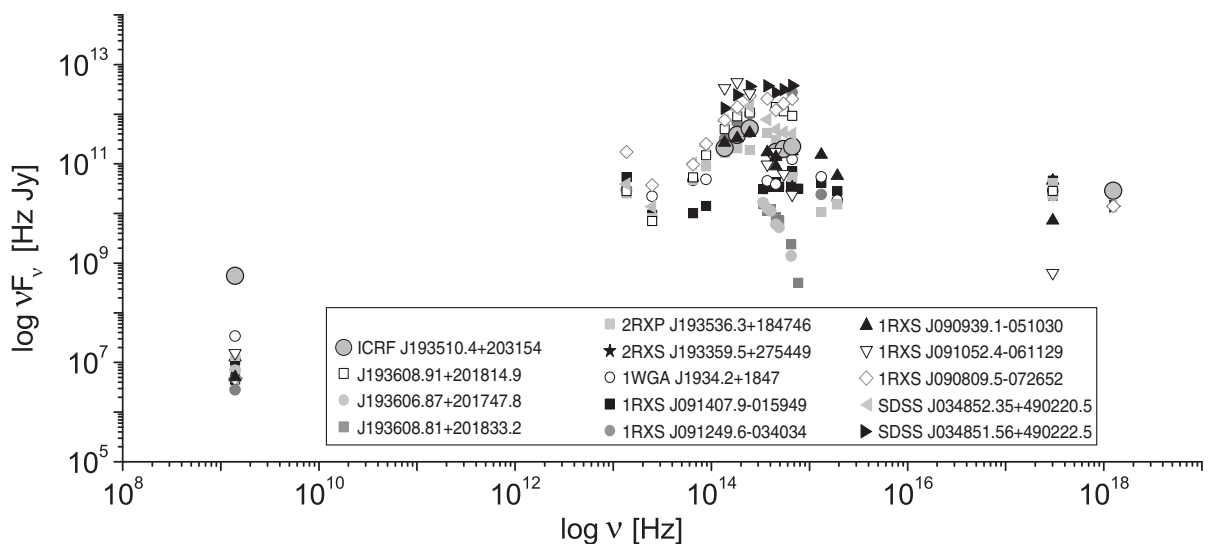

Figure 2. Spectra of 14 pre-selected candidates with a hard component. The object names are specified in the box. The larger grey circles indicate the only source among the 14 that has a $\gamma$-ray component.

After this pre-selection, the sample of candidates consisted of 94 objects, including several multiple identifications of a single source, which were eliminated at the later stages of analysis. We then proceeded to eliminate the candidates using the following criteria. First, we checked the sample for artifacts and crossed off the list all sources with unreliable photometry, as well as extended and polarized sources. Several sources with clearly stellar, galactic, and quasar spectra, as well as spectra which clearly showed lines were also eliminated. The GAIA DR2 database allowed us to use accurate parallax (distance) and proper motion measurements, further restricting the sample to contain only sources with distances 


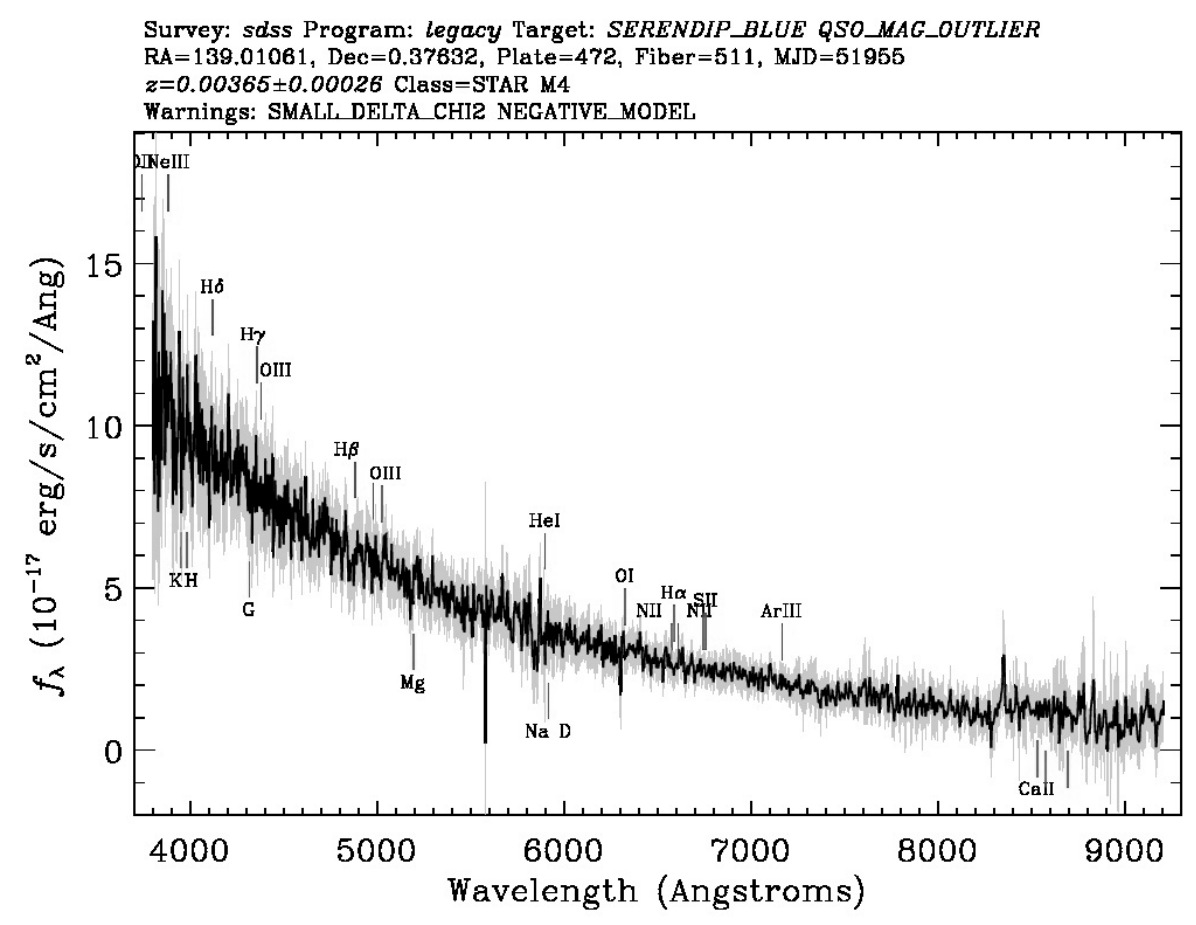

Figure 3. SDSS spectrum of a candidate classified as a DC-type dwarf.

and velocities which would place them inside the (3D) birth location boundary. We then discarded objects with no measured proper motions or proper motions with a large error, as such sources cannot be reliably considered as galactic. The remaining sources were faint and had no spectral data available, and we therefore used SDSS ugriz, BVR, or JHK colors depending on the available photometry to classify them. For well-measured sources, we simply used a Planck curve fit to eliminate stars. For sources with only a few points measured, we used color-color diagrams to keep only the sources located primarily in the regions dominated by white dwarfs and quasars. After this procedure, we were left with a list of 12 candidates. None of them have variability measurements. Four of these candidates have insufficient data available to reject them, and we therefore keep them on the list for now. For the remaining 8 sources, we used the relation described by Beskin \& Karpov (2005), specifically, formula (77) in that paper, to compute the theoretical luminosities expected for a stellar-mass BH located at the point in space where the candidates are. We took into account the ISM densities (calculated using the 3D distribution of dust in the galaxy (Green et al., 2018) and the total column density of interstellar hydrogen), distances and proper motions from GAIA DR2. Using theoretical BH masses and space velocities estimated 
from proper motions, we computed a grid of magnitudes possible for a $\mathrm{BH}$ located in this region of the galaxy. After comparing these computed magnitudes with those of the candidates, we rejected a further 3 sources. The remaining 5 sources fit the luminosity criteria: J035738.16+525934.4, J035757.63+525928.7, $\mathrm{J} 193559.9+205305.7, \mathrm{~J} 035717.10+511525.4, \mathrm{~J} 035239.08+513344.1$. These are all faint sources, $m_{V} \sim 19-21$, located within $300 \mathrm{pc}$. Two of them have hard components. Figure 4 shows the computed magnitude distributions for BHs located at the positions of these 5 sources. These candidates, as well as the 4 with insufficient data for rejection were marked for further investigation. The subject of our future work would involve obtaining spectra for these sources and checking their variability.
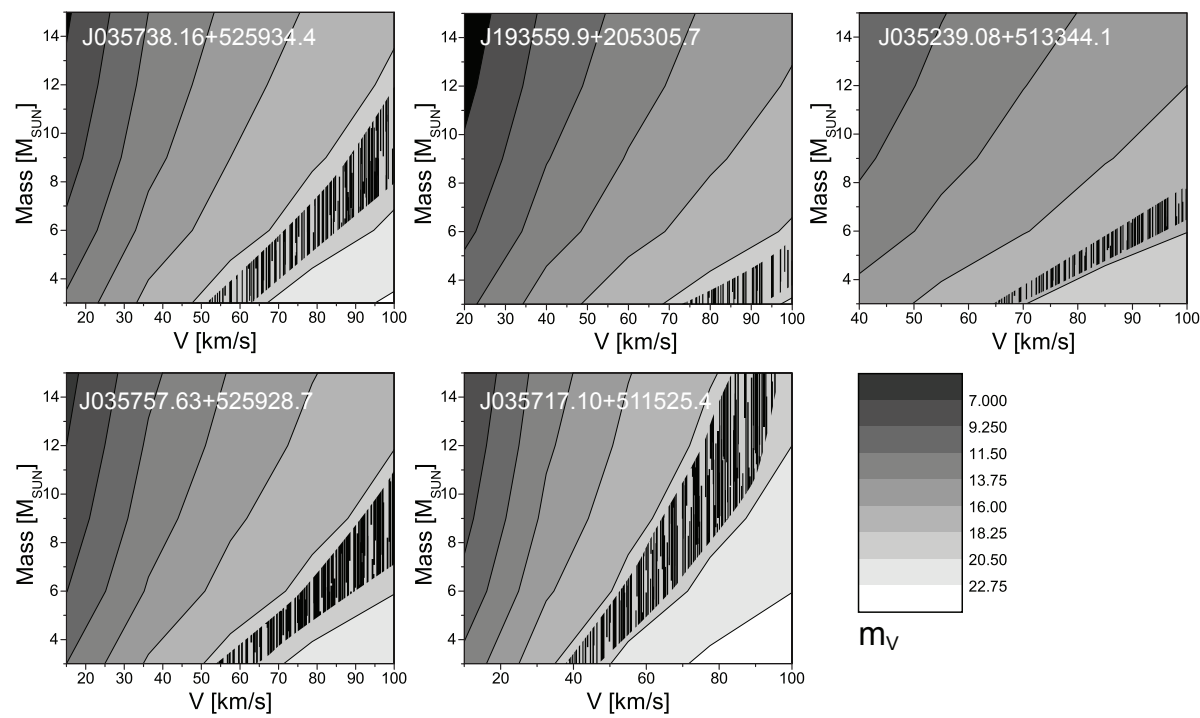

Figure 4. Theoretical magnitude distribution for BHs located at the positions of the 5 candidates. The positions of these sources are marked by black. The name of the object is indicated at the top of each graph in white.

In the second part of our work, now in progress, we seek $\mathrm{BH}$ candidates throughout the whole sky, not restricted to specific sky regions. We now concentrate on objects for which the spectra are already measured and publicly available. Databases like SDSS, LAMOST, and others, have a number of objects whose spectra are marked as "unknown" due to either a lack of spectral features, or poor quality. Some examples of such spectra are shown in Figure 
5. These sources and others with similar featureless spectra are of primary interest to us, especially if they have counterparts in other spectral domains. A high signal-to-noise ratio is preferred in order to rule out the presence of lines with equivalent widths $>5 \AA$. Therefore, DC-type white dwarfs, unconfirmed BL Lacertae objects, and sources of unidentified spectral type with no significant spectral features are our primary objects of interest in the corresponding catalogs. Such sources will be analyzed further using the method described above in order to refine their classification. Those among them that are shown to fit the other selection criteria may be considered as BH candidates. These results will be published in our upcoming paper.
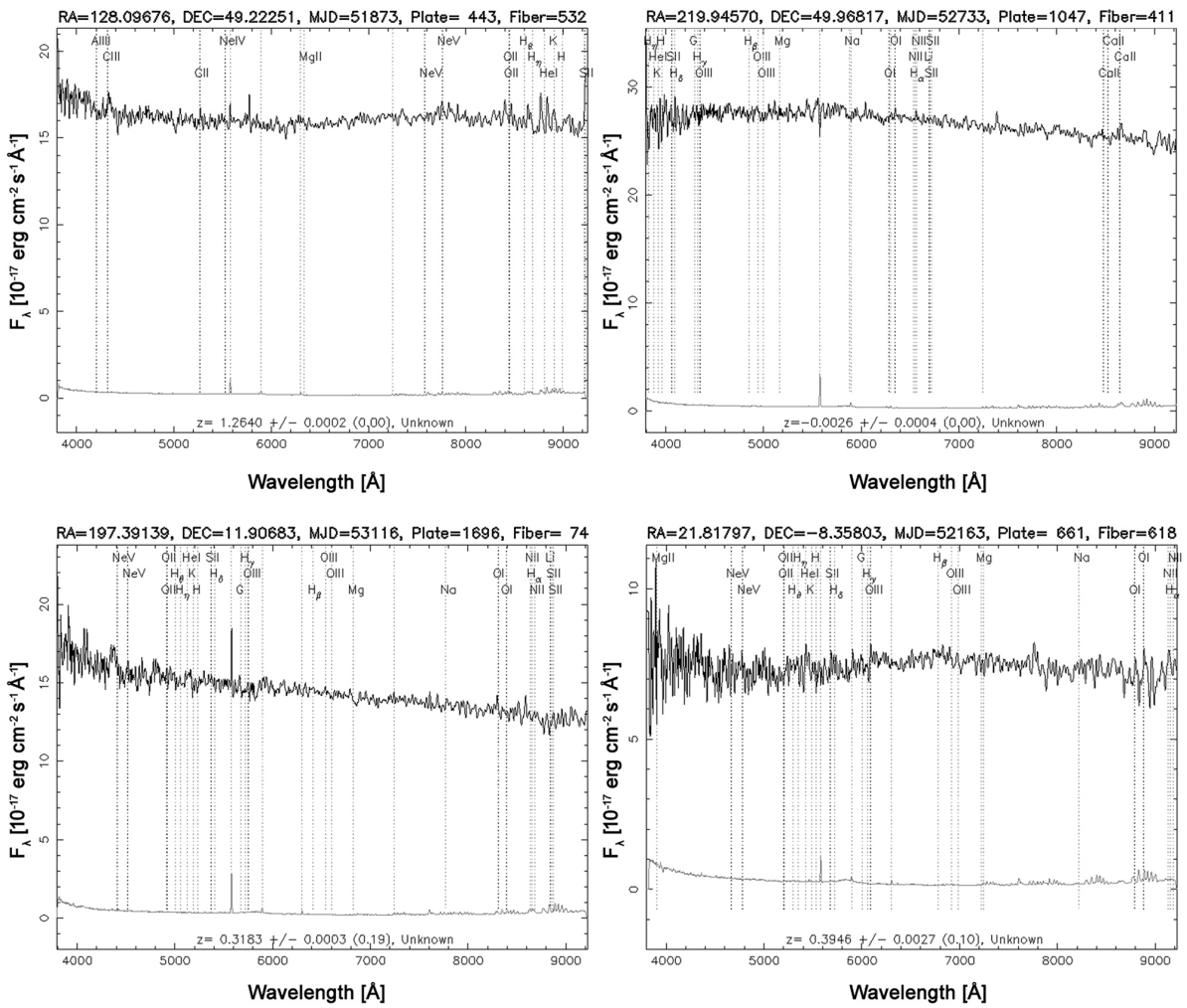

Figure 5. Examples of unidentified spectra in the SDSS. 


\section{Results}

We have performed a search for stellar-mass BH candidates. Looking for BHs is an important task directly related to the problem of direct detection of $\mathrm{BH}$ event horizons, for which they can be viewed as ideal probes. Many now-isolated $\mathrm{BHs}$ may have originated in high mass binaries where the other component is a neutron star. Tracing the trajectories of the neutron stars to their birth places, we placed restrictions on the locations of possible $\mathrm{BH}$ candidates, deriving four promising regions in the sky for the search.

Within those areas, we selected all sources with a hard spectral component, and identified them with their optical counterparts using the SDSS database. Blue objects and DC-dwarfs were also considered. A list of 94 candidates was made in the process, from which we eliminated all sources with unreliable photometry, as well as extended and polarized sources. The several objects with available spectra were also crossed off the list due to their clearly stellar, galactic, or quasar nature. We then used the accurate parallax and proper motion data from the GAIA database to further restrict the sample to objects located within the considered spatial region. Multi-band photometric data were used to classify the remaining sources, thereby shortening the list down to 12 objects.

Theoretical luminosities were then computed for stellar-mass BHs located at the positions of the candidates. The theoretical magnitudes were compared with the observed ones, which allowed us to finalize the preliminary list of BH candidates which fit the observational criteria. There are 5 sources on the list; their coordinates are as follows $(\alpha, \delta): 03: 57: 38.19+52: 59: 34.4,03: 57: 57.64+52: 59: 29.1$, $19: 35: 59.92+20: 53: 07.0,03: 57: 17.10+51: 15: 25.4,03: 52: 39.09+51: 33: 44.1$. Longterm observations of these sources are planned as the next step to narrow the list down further, both to obtain their spectra and monitor them for photometric variations (no spectra or variability information are currently available for these objects). Such observations would allow us to obtain evidence that one or more of these sources are, in fact, possible BHs.

\section{Conclusions}

We have performed a search for stellar-mass black hole candidates within 4 restricted regions of space, computed in the assumption that $\mathrm{BHs}$ have originally formed in high-mass binaries. The search in these areas yielded a list of 5 candidates, J035738.16+525934.4, J035757.63+525928.7, J193559.9+205305.7,

J035717.10+511525.4, and J035239.08+513344.1, which may be considered as a preliminary result. These 5 sources were targeted for further investigation, and will be observed in order to obtain their spectra, as well as data on their variability. This result shows that at least several sources fit the selection criteria (are consistent with the theoretical expectations of a stellar mass black hole as observed from Earth), and that a full-sky search will provide many more 
candidates. This full-sky search, as well as obtaining observed data for the 5 candidates, is the subject of our upcoming work.

\section{References}

Batten, A. H. 1967, Ann. Rev. Astron. Astrophys., 5, 25

Beskin, G. M. \& Karpov, S. V. 2005, Astron. Astrophys., 440, 223

Bethe, H. A. \& Brown, G. E. 1998, Astrophys. J., 506, 780

Bisnovatyi-Kogan, G. S. \& Ruzmaikin, A. A. 1974, Astrophys. Space Sci., 28, 45

Bondi, H. 1952, Mon. Not. R. Astron. Soc., 112, 195

Chisholm, J. R., Dodelson, S., \& Kolb, E. W. 2003, Astrophys. J., 596, 437

Chmyreva, E. G., Beskin, G. M., \& Biryukov, A. V. 2010, Astronomy Letters, 36, 116

Duquennoy, A. \& Mayor, M. 1991, Astron. Astrophys., 500, 337

Green, G. M., Schlafly, E. F., Finkbeiner, D., et al. 2018, Mon. Not. R. Astron. Soc., 478, 651

Halbwachs, J. L., Mayor, M., Udry, S., \& Arenou, F. 2003, Astron. Astrophys., 397, 159

Heckler, A. F. \& Kolb, E. W. 1996, Astrophys. J., Lett., 472, L85

Hobbs, G., Lorimer, D. R., Lyne, A. G., \& Kramer, M. 2005, Mon. Not. R. Astron. Soc., 360, 974

Ipser, J. R. \& Price, R. H. 1982, Astrophys. J., 255, 654

McDowell, J. 1985, Mon. Not. R. Astron. Soc., 217, 77

Meszaros, P. 1975, Astron. Astrophys., 44, 59

Novikov, I. D. \& Thorne, K. S., Black holes (Les Astres Occlus), Edited by C. DeWitt and B. DeWitt, Gordon and Breach, N.Y. 1973, 343-450

Prokhorov, M. E. \& Popov, S. B. 2002, Astronomy Letters, 28, 536

Shapiro, S. L. 1973, Astrophys. J., 180, 531

Shvartsman, V. F. 1971, Soviet Astron, 15, 377

Zeldovich, Y. B. \& Novikov, I. D., Relativistic astrophysics. Vol.1: Stars and relativity, University of Chicago Press. 1971 